\title{
Short-Term Postoperative Outcome of Laparoscopic Total Mesorectal Excision in Rectal Carcinoma
}

\author{
Roy $\mathrm{S}^{1}$, Mondal $\mathrm{SK}^{2}$
}

\begin{abstract}
Despite the proven benefits, laparoscopic colorectal surgery is still under utilized among surgeons. Aim of this study is to determine the feasibility and morbidity after laparoscopic Total Mesorectal Excision(TME), implementing a standardized operative technique and recovery protocol. The first 30 patients treated laparoscopically were included. Standardized oncological clearance ensured by early central vascular ligation, medial to lateral approach, and complete mesorectalexcision. Recovery parameters, short-term outcomes, morbidity and mortality have been assessed.Total number of patient was 30. In 26 patients anterior resection was done, in 4 patients Abdominoperineal resection was done.Overall mean operating time was 224( 242-137) minutes. In anterior resection Mean operating time was 187( 236-137) minutes, whereas in lap APR operating time was 210(242-178) minutes. In total 4 patients(13\%) were converted to open procedure. Average bleeding in anterior resection group was $120 \mathrm{ml}$ (50-200ml), in Lap APR $210 \mathrm{ml}(115-350 \mathrm{ml})$. Mean number of lymphnodes removed in AR 16. In APR1, Mean time to flatus was 1.5 days. Mean time to stool 4.2 days, mean length of hospital stay in AR 8.6 days, in APR 11.5 days. Overall morbidity 23\%(7), major morbidity 10\%(3). There is no mortality, no anastomotic leak, and no 30 days readmission.laparoscopic Total Mesorectal Excision is safe and leads to excellent results in terms of recovery and short term outcomes. Key factors for better outcomes is adoption of a standardized technique and training model.
\end{abstract}

Key words: Total Mesorectal Excision, Anterior resection, Abdominoperineal resection.

1. Corresponding Author: Dr. Sharmistha Roy Assistant Professor, Department of Surgery BIRDEM General Hospital \& IMC e-mail:sprmista@yahoo.com

2. Dr. Samiron Kumar Mondal Associate Professor, Department of Surgery BIRDEM General Hospital \& IMC

\section{Introduction}

Laparoscopic surgery is now well established as a treatment of choice for several malignant and benign colon and rectal disease. Even though laparoscopic Gallbladder surgery has been well established as gold standard, it took several years and many clinical trials to establish the role of laparoscopic surgery in colorectal malignancy. The oncologic clearance and outcome, being the main issue under evaluation and formed the main basis of many international clinical trials investigating rigorously the feasibility of laparoscopic colorectal surgery, cancer risk, morbidity, and recovery benefits. Several large prospective Randomized Controlled trial have been completed and have reported on both short and long term outcomes confirming the efficacy and oncological safety of lap colorectal surgery ${ }^{1.9}$. Despite proven benefits of laparoscopic colorectal surgery, it is still underutilized among general \& colorectal surgeons. The aim of this present study is to determine the feasibility and safety of laparoscopic surgery in rectal malignancies in a learning curve setting. A well-structured training model is adopted. Standardized postoperative recovery protocol followed. Short-term outcome has been recorded including operativedata, recovery parameters and 30 days mortality morbidity.

\section{Material \& Methods}

A prospective database is maintained for first 30 consecutive patients attending our unit with rectal malignancy who does not have the exclusion criteria were registered for this study. Exclusion Criteria were $\mathrm{T}_{4}$ and bulky tumor, BMI $>35$, previous abdominal surgery, Severe cardiopulmonary compromised patient(ASA III), emergency patients with intestinal obstruction or perforation ( in an attempt to take colonoscopic biopsyone patient).Study period was from February 2010 to September 2013 (32months).

Operative data included- Operating time, amount of bleeding, number of lymph node removed with specimen, oncological clearance of resection margins, conversion rate and reason of conversion. Following recovery endpoints are considered to asses short term post operative outcome- Mean time to -passage of flatus, passage of solid stool, start of oral feeding, removal of catheter, removal of drain,independent walk, quit injectable analgesics, days of hospital stay.30 days mortality morbidity assessed by collecting data regarding - 
wound infection, deep vein thrombosis, respiratory complication, cardiac complication, urinary complication, anastomotic failure evident by pelvic drain, Need to readmit within 30 POD or reopen due to complication.

Our series: 30 patients of rectal malignancy have been operated laparoscopically. Among them 9 female \& 21 Male patient. 12 patients were $<50$ years of age(19-49), 18 were $>50(50-78$ ) years. 10 patient had BMI $<25$ ( 2 female, 8 male), others had BMI 25-35(7female, 13 male). BMI more than 35 were not selected for laparoscopic TME.

11 patients had growth near rectosigmoid junction and were selected for laparoscopic anterior resection(AR). 15 patient had growth in rectum $>4 \mathrm{~cm}$ from dentate line, they were also selected for anterior resection. 4 patient had growth $<4 \mathrm{~cm}$ from dentate line, they underwent laparoscopic Abdominoperineal resection (APR).

\section{Results}

The overall mean operating time was 224( 242-137) minutes. In anterior resection Mean operating time was 187( 236-137) minutes, whereas in lap APR operating time was more 210(242-178) minutes. In total 4 patient was converted to open procedure, among them 3 in anterior resection group and 1 in Lap APR group. Reason of conversion was omental adhesion and bleeding in 2, bleeding from inferior mesenteric artery1, tumor adherent to prostate capsule in 1 patient in lap APR. Average bleeding in anterior resection group was $120 \mathrm{ml}(50-$ 200ml), in Lap APR $210 \mathrm{ml}(115-350 \mathrm{ml})$. Post operative recovery period data showed, in anterior resection first flatus passed within 1-3 days, whereas in APR group colostomy bag inflated with bowel gas in $2 \pm 1$ days. Overall mean time to passage of flatus was 1.5 day.Passage of solid stool in AR 6 \pm 2 (4-8POD) days, in APR stool in colostomy bag in $2 \pm 1$ (1-3POD). Mean time to passage of solid stool was 4.2 days.Sips of water allowed to all patients from $1^{\text {st }}$ POD. Solid food was given in AR group on $4^{\text {th }}-6^{\text {th }} \mathrm{POD}(5+/-1$ days $)$ as abdominal conditions permit, in APR group solid oral diet was given earlier as there was no anastomosis, which was as early as $2^{\text {nd }}$ POD. Drain was taken out once oral diet started and stool passed in AR group which was $6 \pm 2$ days. In APR drain removed when it was $<20 \mathrm{ml}$ in 24 hour, which was $5 \pm 1$ days.Urinary catheter was removed in all AR patients on $2^{\text {nd }}$ POD to allow early mobilization, in APR group Catheter was kept longer( $7 \pm 3$ POD) as it was difficult to mobilize early with the perineal wound.In AR group patient could walk around independently in 2nd$4^{\text {th }} \mathrm{POD}(3 \pm 1$ days $)$, in APR group it was

$6^{\text {th }}-12^{\text {th }} \operatorname{POD}(9 \pm 3$ days $)$. Patients were discharged when they were taking solid food, has passed solid stool, could walk independently, and free of injectable analgesics. In AR group mean hospital stay was 9.6 days(8-11 days) after surgery, in APR group it was 13days(10-16days) after surgery. 30 days post operative mortality and morbidity was recorded during theirfollow up. 2 patients developed chest infection in early postoperative period, and improved with chest physiotherapy and mobilization, 1 patient with pre-existing ischemic heart disease developed heart failure and pulmonary edema due to fluid overload and was treated with medication. 2 patients developed minor wound infection and improved with bedside dressing. 2 patient developed urinary incontinence after removal of catheter. There was documentedurinary tract infection, and antibiotic given according to culture sensitivity. Detrusor muscle stabilizer also used in one case to control the situation. There was no incidence of DVT, readmission, reoperation, and no mortality.

Table- I: Patient Profile

\begin{tabular}{ccccccc}
\hline Gender & \multicolumn{2}{c}{$\begin{array}{c}\text { Number of } \\
\text { case (\%) }\end{array}$} & Age $<$ 50yrs & Age $>$ 50yrs & BMI < 25 & BMI 25-35 \\
Male & 21 & $(70 \%)$ & 7 & 14 & 8 & 13 \\
Female & 9 & $(30 \%)$ & 3 & 6 & 2 & 7 \\
\hline
\end{tabular}

Table-II: Diagnosis

\begin{tabular}{cccc}
\hline Site of lesion & Number & $\begin{array}{c}\text { Stage }{ }^{*}(\text { number of } \\
\text { cases) }\end{array}$ & Operation \\
\hline Growth near & 11 & $\mathrm{PT}_{2} \mathrm{~N}_{0}(4)$ & Anterior resection \\
rectosigmoid & & $\mathrm{P} \mathrm{T}_{2} \mathrm{~N}_{1}(3)$ & \\
junction & & $\mathrm{P} \mathrm{T}_{3} \mathrm{~N}_{1}(4)$ & \\
Growth in rectum & 15 & $\mathrm{PT}_{1} \mathrm{~N}_{0}(2)$ & Anterior resection \\
$>4$ cm from dentate & & $\mathrm{P} \mathrm{T}_{2} \mathrm{~N}_{0}(5)$ & \\
line & & $\mathrm{P} \mathrm{T}_{2} \mathrm{~N}_{1}(6)$ & \\
& & $\mathrm{P} \mathrm{T}_{3 \mathrm{a}} \mathrm{N}_{1}(2)$ & \\
Growth<4cm from & 4 & $\mathrm{P} \mathrm{T}_{3} \mathrm{~N}_{0}(1)$ & Abdominoperineal \\
dentate line & & $\mathrm{P} \mathrm{T}_{2} \mathrm{~N}_{1}(3)$ & resection \\
\hline
\end{tabular}

* Staging is done as per American Joint Committee on Cancer (AJCC) TNM Staging .

Table-III: Operative data

\begin{tabular}{ccccc}
\hline & Time(minutes) & $\begin{array}{c}\text { Number of } \\
\text { lymphnodes } \\
\text { removed }\end{array}$ & Conversion & $\begin{array}{c}\text { Bleeding } \\
(\mathbf{m l})\end{array}$ \\
\hline Over all & $224(242-137)$ & $13(11-17)$ & 4 & $150(50-350)$ \\
Anterior resection & $187(236-137)$ & $16(13-17)$ & 3 & $120(50-200)$ \\
$\begin{array}{c}\text { Abdominoperineal } \\
\text { resection }\end{array}$ & $210(242-178)$ & $15(11-16)$ & 1 & $210(115-350)$ \\
\hline
\end{tabular}

Table-IV: Post operative recovery

\begin{tabular}{ccc}
\hline Recovery end points & $\begin{array}{c}\text { Anterior resection } \\
\text { Mean }+\mathbf{S D}^{*} \text { (days) }\end{array}$ & $\begin{array}{c}\text { Abdominoperineal resection } \\
\text { Mean }+ \text { SD (days) }\end{array}$ \\
\hline Passage of flatus & $2 \pm 1$ & $2 \pm 1$ \\
Oral feeding & $5 \pm 1$ & $2 \pm 1$ \\
Independent movement & $3 \pm 1$ & $9 \pm 3$ \\
Catheter removed & $2^{\text {nd }}$ POD & $7 \pm 3$ \\
Drain removed & $6 \pm 2$ & $5 \pm 1$ \\
I/V analgesics stopped & $4 \pm 1$ & $5 \pm 2$ \\
Passage of solid stool & $6 \pm 2$ & $2 \pm 1$ \\
Discharge & $9.6 \pm 1.4$ & $13 \pm 3$ \\
\hline
\end{tabular}

SD- Standard Deviation. 
Table-V: Mortality \& morbidity

\begin{tabular}{ccc}
\hline Complication & Number(30) & Percent $\mathbf{1 0 0 \%}$ \\
\hline Pulmonary & 2 & $6.6 \%$ \\
Cardiac & 1 & $3.3 \%$ \\
Wound infection & & $6.6 \%$ \\
Perianal wound in APR & 1 & \\
Abdominal wound in AR & 1 & \\
Urinary incontenence & 2 & $6.6 \%$ \\
Death & 0 & $0 \%$ \\
Re admission/ reoperation & 0 & $0 \%$ \\
\hline
\end{tabular}

\section{Discussion}

The technique of TME for the treatment of rectal carcinoma is increasingly recognized as the new benchmark of quality. One of the most controversial areas of laparoscopic surgery has been laparoscopic resection of rectal cancer, the main concerns being adequate oncological clearance ie quality of TME inlaparoscopic technique. The driver of laparoscopic surgery in the setting of carcinoma rectum, was clearly the possibility of gaining patient benefits. It was presumed, but not proven, that a shorter incision would result in less pain, short duration of ileus, and shorter hospital stay and post operativerecovery. Data on recovery endpoints are now available, demonstrating the main advantages of laparoscopic approach: length of hospital stay, duration of ileus and duration of analgesic use or postoperative pain. Short-term complications, morbidity and mortality were investigated and found to be very similar between groups in all the trials. ${ }^{1-9}$

Our study regarding feasibility of starting laparoscopic total mesorectal excision in carcinoma rectum in a learning curve of laparoscopic colorectal surgery shows encouraging results. We have been selective in choosing patients for laparoscopic surgery in our initial phase, to avoid too much difficulty per operatively and thus surgeon's frustration. The mean operating time was 224 minutes in our study, which is acceptable but slightly more than other studies showing results of laparoscopic TME. ${ }^{10}$ Per operative bleeding in APR group was more due to perineal dissection, which remains same in open surgery. So no additional bleeding was encountered due to adopting laparoscopic technique. But in AR group per operative bleeding was very small 50-200 ml. ${ }^{11}$ The number of lymphnodes removed with the specimen was 13-17 in anterior resection group, average 16. In APR group number of lymphnodes were 11-16, average 15 . This is an indirect evidence of adequacy of laparoscopic mesorectal excision. Postoperativerecoverieswere prompt. Less ileus resulted in early return of bowel sound, so oral diet could be started as early as $1^{\text {st }}$ POD in APR group. Less injectable analgesics were required as incision was smaller. Patients of anterior resection could walk with out support as early as $2^{\text {nd }}$ POD. There was no mortality, no readmission or necessity of reoperation in 30 days postoperative. 2 patient developed chest complication, but nothing major. 2 minor wound infection occurred one in perineal wound that cannot be attributed to laparoscopic surgery, and one in the abdominal wound through which AR resected specimen was delivered. 2 patient (6.6\%)developed incontinence due to urinary tract infection after laparoscopic TME, which was comparable to standard of such complication in open TME in standard colorectal centers. ${ }^{12}$

In conclusion, the potential advantageof laparoscopic TME in rectal carcinoma is less bleeding and magnified view allowing precise dissection of pelvic autonomics. Post operative benefits being less pain, less metabolic response to trauma, early return of gut activity, and improved cosmesis. Potential disadvantages are crowding of instruments in pelvis, fume can obscure the vision, retraction of rectum can be difficult, division of rectum can be difficult, steep learning curve, and increased operating time. The true assessment of safety of Laparoscopic TME in malignancy must come from long termfollowup studies of patients operated by this technique. Currently ongoing such studies will provide evidence-based data on cancer free survival in near future.

\section{Reference}

1. Lacy AM, Garcia-Valdecasas JC, Delgado S. Laparoscopy-assisted colec-tomy versus open colectomy for treatment of non-metastatic colon cancer: a randomised trial. Lancet. 2002;359(9325):2224e9.

2. Lacy AM, Delgado S, Castells A. The long-term results of a randomized clinical trial of laparoscopy-assisted versus open surgery for colon cancer. Ann Surg. 2008;248(1):1e7.

3. A comparison of laparoscopically assisted and open colectomy for colon cancer. N Engl J Med.2004;350(20):2050e9.

4.FleshmanJ,SargentDJ,GreenE.Laparoscopiccolectomyfo rcancerisnot inferior to open surgery based on 5-year data from the COST study group trial. Ann Surg. 2007;246(4):654-5.

5.Veldkamp R, Kuhry E, Hop WC. Laparoscopic surgery versus open surgery for colon cancer: short-term outcomes of a randomised trial. Lancet Oncol.2005;6(7):477-84.

6.Buunen M, Veldkamp R, Hop WC. Survival after laparoscopic surgery versus open surgery for colon cancer: long-term outcome of a randomised clinical trial. Lancet Oncol. 2009;10(1):44-52.

7.Guillou PJ, Quirke P, Thorpe H. Short-term endpoints of conventional versus laparoscopic-assisted surgery in patients with colorectal cancer (MRC CLASICC trial): multicentre, randomised controlled trial. Lancet.2005;365(9472):1718-26.

8.JayneDG,GuillouPJ,ThorpeH.Randomizedtrialoflaparosc opic-assisted resection of colorectal carcinoma: 3-year results of the UK MRC CLASICC trial Group. 


\section{J ClinOncol.2007;25(21):3061-8.}

9.Luglio G, Nelson H. Laparoscopy for colon cancer: state of the art. Surg. Oncol. Clin N Am.2010;19(4):777-91.

10.Luglio G, G Domenico, De Palma, R Tarquini. Laparoscopic colorectal surgery in learning curve: Role of implementation of a standardized technique and recovery protocol, A cohort study.Annals of medicine and surgery. 2015;4:89-94.
11. Milsom JW, Bohm B, Hammerhofer KA, Fazio V, Steiger E, Elson P. A prospective, randomized trial comparing laparoscopic versus conventional techniques in colorectal cancer surgery: a preliminary report. J Am CollSurg. 1998;187:46-57.

12.Schwenk W, Haase O, Neudecker JJ, Müller JM. Short term benefits for laparoscopic colorectal resection. Cochrane Database of Systematic Reviews 2005, Issue 2. Art. No.: CD003145. DOI: 10.1002/14651858.CD003145.pub2. 